\title{
Ovarian cancer stem cells: A target for oncological therapy
}

\author{
Anna Markowska ${ }^{1, A, D}$, Stefan Sajdak ${ }^{2, E}$, Adam Huczyński ${ }^{3, D, E}$, Sandra Rehlis ${ }^{4, B}$, Janina Markowska ${ }^{5, D-F}$ \\ ${ }^{1}$ Department of Perinatology and Women's Diseases, Poznan University of Medical Sciences, Poland \\ 2 Department of Gynecological Surgery, Poznan University of Medical Sciences, Poland \\ ${ }^{3}$ Faculty of Chemistry, Adam Mickiewicz University in Poznań, Poland \\ ${ }^{4}$ Klinikum Fulda, Universitätsmedizin, Marburg, Germany \\ ${ }^{5}$ Department of Oncology, Poznan University of Medical Sciences, Poland \\ A - research concept and design; $B$ - collection and/or assembly of data; $C$ - data analysis and interpretation; \\ $D$ - writing the article; $E$ - critical revision of the article; $F$ - final approval of the article
}

Address for correspondence

Anna Markowska

E-mail:annamarkowska@vp.pl

\section{Funding sources}

None declared

\section{Conflict of interest}

None declared

Received on January 11, 2017

Reviewed on April 2, 2017

Accepted on May 23, 2017

\begin{abstract}
According to numerous studies, failures in treatment of ovarian cancer, i.e., a relapse and metastases, result from a small population of cancer stem cells (CSCS). They may also be responsible for tumor initiation. Cancer stem cells are resistant to chemo- and radiotherapy. Eradication of CSCS may involve the application of salinomycin, metformin and Clostridium perfringens; the effect of anti-angiogenic factors remains controversial. Salinomycin is an antibiotic isolated from Streptomyces albus bacteria. Its CSC-eradicating effect has been demonstrated both in ovarian cancer cell lines and in women with breast cancer. Clostridium perffringens enterotoxin (CPE) has been demonstrated to destroy CSCs in ovarian cancer both in vivo and in vitro. Metformin, apart from its hypoglycemic effect, reduces the CSC population and inhibits the proliferation of neoplastic cells and angiogenesis. Cancer stem cells with expression of VEGFR1+ have been described as affecting circulating cancer cells and influencing the formation of metastases. Both positive and negative effects of anti-angiogenic therapy on the CSC population have been documented.
\end{abstract}

Key words: angiogenesis, metformin, salinomycin, ovarian cancer stem cells, Clostridium perfringens enterotoxin

DOI

10.17219/acem/73999

\section{Copyright}

Copyright by Author(s)

This is an article distributed under the terms of the

Creative Commons Attribution Non-Commercial License

(http://creativecommons.org/licenses/by-nc-nd/4.0/) 
Among all malignant gynecological tumors, ovarian cancer represents the leading cause of death. The majority of patients are diagnosed with advanced stages. Standard treatment for ovarian cancer is primary maximum cytoreductive surgery followed by chemotherapy with carboplatin and paclitaxel. After initial debulking and first line chemotherapy, around $50-70 \%$ of patients reach full clinical response. However, in $80 \%$ of patients this is followed by a relapse of the disease, the cause of therapeutic failure. ${ }^{1,2}$

According to numerous studies, the principal cause of the cancer relapse and metastases stems from a small cell population resistant to chemo- and radiotherapy, comprising less than 2-5\% of tumor mass and involving cancer stem cells (CSCs). Most probably, this specific cell subpopulation is also linked to the initiation of the tumor (cancer initiating cells - CICs). ${ }^{1,3-6}$

Cancer stem cells manifest the capacity of self-renewal, DNA repair, persisting in the G1 or G0 cell cycle phases as inactive dormant cells and asymmetric cell division. They do not undergo apoptosis and they manifest overexpression of $A B C$ genes, which is linked to their resistance to cytostatic drugs. Control of their self-replacement is associated in principle with numerous signaling pathways, including Notch, Sonic hedgehog (Shh) and Wnt. ${ }^{2,7,8}$

Cancer stem cells can be identified and isolated due to their specific markers, such as CD44, CD133 (prominin-1), CD117 (c-Kit), ALDH1 (aldehyde dehydrogenase), and OCT3/4 (POU5F1), the transcription factor of the POU family. ${ }^{9-11}$

In 2010, CSCs were isolated and identified in ovarian cancer. ${ }^{10}$ In 2014, in in vivo and in vitro conditions, cisplatin was demonstrated to inhibit both the formation of metastases and the invasion of CICs manifesting the marker of CD $/ 44^{+} / \mathrm{CD} 117^{+}$, but only those with overexpression of CXCR4. Thus, cisplatin inhibited the activity of the SDF-1/CXCR4 axis (the factor originating from stromal cells and its ligand). ${ }^{12}$

Using an animal model, Abubaker et al. demonstrated that short-term application of chemotherapy (cisplatin and paclitaxel) enriched the cell population with high expression of Oct- 4 and CD117. ${ }^{13}$ Similar experiments were performed by Zhou et al. ${ }^{14}$ Following the administration of cisplatin, the dormant cells of human ovarian cancer manifested an augmented expression of cells with Oct-4, nestin, CD-117, and CD44 markers, proving the resistance of this stem cell fraction to cisplatin.

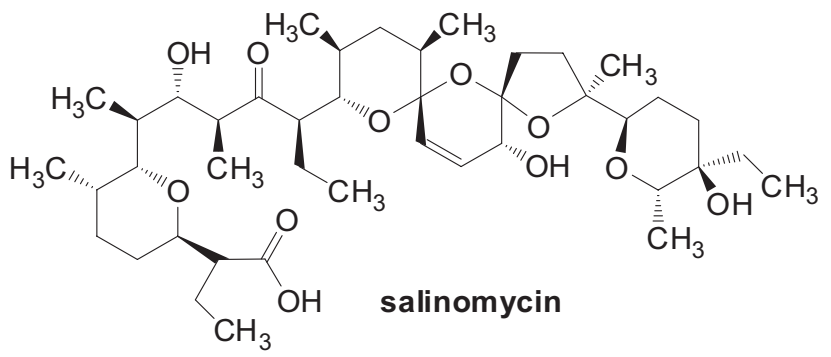

Both the CSC population with its typical markers and the signaling pathways that CSCs take advantage of are thought to provide potential targets for therapy. ${ }^{7,8,13}$

According to Massard et al., there are 2 options for eradicating CSCs: one involves the induction of their differentiation, upon which they lose their capacity of selfreplacement (retinoic acid demonstrated such an ability); the other one involves the elimination of CSCs by targeted inhibitors of signaling pathways. ${ }^{3}$

Below, we outline the manners of CSC eradication using salinomycin, metformin and enterotoxin of Clostridium perfringens, and we discuss the controversial effects of anti-angiogenic factors.

\section{Salinomycin}

Isolated in Japan in 1974 from Streptomyces albus, salinomycin represents a carboxylic polyether ionophore antibiotic, leading to disturbances in $\mathrm{Na}^{+} / \mathrm{K}^{+}$ion equilibrium in biological membranes and, due to reduced $\mathrm{K}^{+}$concentration, in mitochondria and cytoplasm, resulting in cell apoptosis. ${ }^{15,16}$ Salinomycin is also active through several other mechanisms in tumors of various locations. It inhibits the conserved Wnt pathway, providing CSCs with the ability to self-replace and resist radiotherapy. Salinomycin also reduces the resistance of CSCs to chemotherapy as it affects the activity of $\mathrm{ABC}$ transporters. It seems that several mechanisms of salinomycin action are currently discussed. ${ }^{5,17-19}$

Studies by Zhang et al. in in vivo and in vitro conditions demonstrated that salinomycin induces apoptosis in the cells of ovarian cancer resistant to cisplatin, apoptosis linked to the pathway of mitogen-activated protein kinase (MAPK). ${ }^{20}$ Studies by Kaplan and Teksen on the stable ovarian cancer cell line (OVCAR-3) demonstrated the activity of salinomycin: $40 \%$ of cells underwent apoptosis within 24 h of exposure. ${ }^{21}$ A decrease was noted in the expression of the anti-apoptotic gene $B c l 2$.

Chung et al., using the OVCAR-3 ovarian cancer cell line, isolated stem cells of markers $\mathrm{CD} 44^{+}$and $\mathrm{CD} 117^{+}$, linked to chemoresistance. ${ }^{22}$ They used paclitaxel + salinomycin. Supplementation with salinomycin significantly inhibited the growth of the cells. Another mechanism of salinomycin activity is linked to the activity of death receptor 5 (DR5) and the activation of apoptosis-initiating caspase 8.<smiles>CN(C)C(=N)NC(=N)N</smiles> 
Binding of DR5 to its ligand leads to alterations in death domain (DD, part of the caspase), mobilizing the enzymatic cascade which results in the death of the cell. ${ }^{23}$

Another mechanism of apoptosis in the ovarian cancer cells resistant to cisplatin involves the inhibitory effect of salinomycin on the nuclear transcription factor NF-kB, which affects the control of several other genes. ${ }^{24}$

In practice, salinomycin is also being used by prof. Cord Naujokat in Heidelberg, Germany, obtaining good results among invasive carcinoma in screening studies. The administration of salinomycin has resulted in the inhibition of disease progress over an extended period of time. It is worth noting that acute side effects were rare and serious long-term adverse side effects were not observed. ${ }^{17}$

It has also been demonstrated that salinomycin is able to kill CSCs, regular tumor cells and highly indolent tumor cells displaying resistance to cytotoxic drugs, radiation and the induction of apoptosis. ${ }^{16}$

Up to now, the results obtained from in vivo studies on human xenograft mice and also clinical studies have revealed that salinomycin is able to effectively eliminate $\mathrm{CSCs}$ and to induce partial clinical regression in therapyresistant cancers. ${ }^{17}$

\section{Metformin}

Metformin represents a drug of the biguanide group, used orally in patients with diabetes type II, particularly in overweight/obese patients. Population studies have proved that metformin-treated patients manifested a lowered risk of malignant tumors in several locations, including the preventive effect of the drug in ovarian cancer. In comparison to patients not using metformin, women who develop ovarian cancer and being treated with this drug are older and diagnosed more frequently at earlier International Federation of Gynecology and Obstetrics (FIGO) clinical stages. In the course of the disease, these women manifest longer duration to progression and display a higher 5 -year survival percentage. ${ }^{25-28}$

Numerous studies have shown that the favorable effect of metformin in the development and course of malignant tumors, including ovarian cancer, may be linked to its inhibitory effect on CSCs. ${ }^{29-31}$

Studies on the SKOV3 and A2780 cell lines of ovarian cancers conducted by Zhang et al. demonstrated that low doses of metformin selectively inhibit CSCs with markers CD44+ and CD117+, and inhibit epithelial mesenchymal transition (EMT). ${ }^{32}$ Epithelial mesenchymal transition is linked to the activity of transcription factors and the activation of several genes, resulting in the inhibition of apoptosis and an increased mobility of cells, linked to metastases.

Shank et al. confirmed better prognosis in ovarian cancer patients treated with metformin. ${ }^{29}$ The authors expressed the opinion that the mechanism of its action involved a reduction in the CSC population with ALDH1+ marker, reduced cell proliferation and inhibited angiogenesis. They argued that the results provided rationale for the application of metformin in patients treated due to ovarian cancer.

\section{Clostridium perfringens enterotoxin}

Natural receptors for Clostridium perfringens enterotoxin (CPE) involve claudins 3 and 4 . Claudins 3 and 4 represent the family of tight junction proteins responsible for the integrity of the cell membrane and for its functions. In ovarian cancer, overexpression of claudins 3 and 4 is noted; they play a critical role in invasion and metastases as well as in resistance to chemotherapy. They may provide an attractive target in the treatment of cancer resistant to platins. ${ }^{33}$

Casagrande et al. described the mechanism through which CPE becomes activated in ovarian cancer. ${ }^{34}$ They examined a small number of ovarian CSCs in vitro and in vivo, carrying the marker of CD44+. They exhibited a high expression of claudin 4 . Following the exposure to CPE, the cells underwent apoptosis. Following a sublethal dose of $\mathrm{CPE}$, ovarian cancer xenografts to mice regressed, resulting in a long survival of the animals. Similar results were described by other authors conducting research on ovarian CSCs, CD44+, resistant to paclitaxel and carboplatin. Clostridium perfringens enterotoxin may represent an unconventional therapy eradicating ovarian cancer CSCs. ${ }^{35}$

\section{How about an anti-angiogenic therapy?}

Bevacizumab, an anti-angiogenic humanized monoclonal antibody, is used in first line therapy in advanced ovarian cancer. According to Rauh-Hain et al., relapses of ovarian cancer in patients treated with bevacizumab were located in other body parts than in patients not treated with the antibody. ${ }^{36}$ Those receiving bevacizumab more frequently developed relapses in the pleura, lungs, brain, liver, and lymph nodes than patients on the anti-angiogenic treatment.

Since relapses, as presented above, were linked to CSC metastases, one can speculate on a link between anti-angiogenic therapy and CSC activity. Kaplan et al. found that CSCs with expression of VEGFR1+ promote chemotaxis and adherence of circulating tumor cells. ${ }^{37}$ VEGFR1+ cells establish specific "premetastatic" niches, forming cellular clusters there before the inflow of tumor cells. The application of the antibody specific to VEGFR1+ or the removal of the cells prevents metastases. In addition, VEGFR1+ cells manifest expression of VLA4 (known as integrin $\alpha_{4 \beta 1}$ ), the ligand of which involves fibronectin taking part in cell 
interaction with the matrix. According to Chau et al., the identification of factors targeted at CSCs and associating them with angiogenesis inhibitors might warrant their better clinical efficacy. ${ }^{38}$ The authors also suggest that antiangiogenic therapy leads to tumor hypoxia, resulting in the growth of the CSC population. In their study, Conley et al. presented a similar hypothesis. ${ }^{39}$ The problem of anti-angiogenic therapy in ovarian cancer and, in particular, its relationship with CSCs, is extremely intriguing. Anti-angiogenic action based on immunotherapy (vaccine) might uncover another relationship with CSCs. ${ }^{40}$ Regarding future directions in the treatment of ovarian cancer, it would seem that combined therapy - cytostatics, anti-angiogenics and anti-CSCs - is a very promising option.

\section{References}

1. Chen J, Wang J, Zhang Y, et al. Observation of ovarian cancer stem cell behavior and investigation of potential mechanisms of drug resistance in three-dimensional cell culture. J Biosci Bioeng. 2014; 118(2):214-222.

2. Shah MM, Landen CHN. Ovarian cancer stem cells: Are they real and why are they important? Review. Gynecol Oncol. 2014;132(2):483-489.

3. Massard C, Deutsch E, Soria JC. Tumor stem cell-targeted treatment: Elimination or differentiation. Ann Oncol. 2006;17(11):1620-1624.

4. Rich JN. Cancer stem cells in radiation resistance. Cancer Res. 2007; 67(19):8980-8984.

5. Shukla G, Srivastava AK, Patidar R, Khara P, Saxen R. Therapeutic potential, challenges and future perspective of cancer stem cells in translational oncology: A critical review. Curr Stem Cell Res Ther. 2017;12(3):207-224

6. Garcia-Rubino ME, Lozano-Lopez C, Campos JM. Inhibitors of cancer stem cells. Anticancer Agents Med Chem. 2016;16(10):1230-1239.

7. Tomao F, Papa A, Rossi L, et al. Emerging role of cancer stem cells in the biology and treatment of ovarian cancer: Basic knowledge and therapeutic possibilities for an innovative approach. J Exp Clin Cancer Res. 2013;32:48.

8. Eyler CE, Rich JN. Survival of the fittest: Cancer stem cells in therapeutic resistance and angiogenesis. J Clin Oncol. 2008;26(17): 2839-2845.

9. Zeng J, Ruan J, Luo L, et al. Molecular portraits of heterogeneity related to cancer stem cells in human ovarian cancer. Int $J$ Gynecol Cancer. 2014;24(1):29-35.

10. Bapat SA. Human ovarian cancer stem cells. Reproduction. 2010;140(1): 33-41.

11. Shi YY, Jiang H. Prognostic role of the cancer stem cell marker CD44 in ovarian cancer: A meta-analysis. Genet Mol Res. 2016;15(3). doi: 10.4238/gmr.15038325

12. Yu Z, Liu T, Zhao Y, et al. Cisplatin targets the stromal cell-derived factor-1-CXC chemokine receptor type 4 axis to suppress metastasis and invasion of ovarian cancer-initiating cells. Tumor Biol. 2014; 35:4637-4644.

13. Abubaker K, Latifi A, Luwor R, et al. Short-term single treatment of chemotherapy results in the enrichment of ovarian cancer stem cell-like cells leading to an increased tumor burden. Mol Cancer. 2013;12:24. doi:10.1186/1476-4598-12-24

14. Zhou N, Wu X, Yang B, Zhang D, Qing G. Stem cell characteristics of dormant cells and cisplatininduced effects on the stemness of epithelial ovarian cancer cells. Mol Med Rep. 2014;10(5):2495-2504.

15. Miyazaki Y, Shibuya M, Sugawara H, Kawaquchi O, Hirsoe C. Salinomycin, a new polyether antibiotic. J Antibiot (Tokyo). 1974;27(11): 814-821.

16. Antoszczak M, Huczyński A. Anticancer activity of polyether ionophore-salinomycin. Anticancer Agents Med Chem. 2015;15(5):575-591.

17. Naujokat $C$, Steinhart R. Salinomycin as a drug for targeting human cancer stem cells.J Biomed Biotechnol. 2012;950658. doi:10.1155/2012/ 950658
18. Fuchs D, Heinold A, Opelz G, Daniel V, Naujokot C. Salinomycin induces apoptosis and overcomes apoptosis resistance in human cancer cells. Biochem Biophys Res Commun. 2009;390(3):743-749.

19. Kim JH, Yoo HI, Kang HS, Ro J, Yoon S. Salinomycin sensitizes antimitotic drugs-treated cancer cells by increasing apoptosis via the prevention of G2 arrest. Biochem Biophys Res Commun. 2012;418(1): 98-103.

20. Zhang B, Wang X, Cai F, Chen W, Loesch U, Zhong XY. Antitumor properties of salinomycin on cisplatin-resistant human ovarian cancer cells in vitro and in vivo: Involvement of p38 MAPK activation. Oncol Rep. 2013;29(4):1371-1378.

21. Kaplan F, Teksen F. Apoptotic effects of salinomycin on human ovarian cancer cell line (OVCAR-3). Tumour Biol. 2016;37(3):3897-3903.

22. Chung H, Kim YH, Kwon M, et al. The effect of salinomycin on ovarian cancer stem-like cells. Obstet Gynecol Sci. 2016;59(4):261-268.

23. Parajuli B, Shin SJ, Kwon SH, et al. Salinomycin induces apoptosis via death receptor-5 up-regulation in cisplatin-resistant ovarian cancer cells. Anticancer Res. 2013;33(4):1457-1462.

24. Parajuli B, Lee HG, Kwon SH, et al. Salinomycin inhibits Akt/NF-KB and induces apoptosis in cisplatin resistant ovarian cancer cells. Cancer Epidemiol. 2013;37(4):512-517.

25. Libby G, Donnelly LA, Donnan PT, Alessi DR, Morris AD, Evans JM. New users of metformin are at low risk of incident cancer: A cohort study among people with type 2 diabetes. Diabetes Care. 2009;32(9): 1620-1625.

26. Dilokthornsakul P, Chaiyakunapruk N, Termrungruanglert W, Pratoomsoot C, Saokaew S, Sruamsiri R. The effects of metformin on ovarian cancer: A systematic review. Int J Gynecol Cancer. 2013;23(9): 1544-1551.

27. Kumar S, Meuter A, Thapa $P$, et al. Metformin intake is associated with better survival in ovarian cancer: A case-control study. Cancer. 2013;119(3):555-562.

28. Romero IL, McCormick A, McEwen KA, et al. Relationship of type II diabetes and metformin use to ovarian cancer progression, survival, and chemosensitivity. Obstet Gynecol. 2012;119(1):61-67.

29. Shank JJ, Yang K, Ghannam J, et al. Metformin targets ovarian cancer stem cells in vitro and in vivo. Gynecol Oncol. 2012;127(2):390-397.

30. Del Barco S, Vazquez-Martin A, Cufí S, et al. Metformin: Multi-faceted protection against cancer. Oncotarget. 2011;2(12):896-917.

31. Charafe-Jauffret E, Monville F, Ginestier C, Dontu G, Birnbaum D, Wicha MS. Cancer stem cells in breast: Current opinion and future challenges. Pathobiology. 2008;75(2):75-84.

32. Zhang $\mathrm{R}$, Zhang $\mathrm{P}$, Wang $\mathrm{H}$, et al. Inhibitory effects of metformin at low concentration on epithelial-mesenchymal transition of CD44(+) CD117(+) ovarian cancer stem cells. Stem Cell Res Ther. 2015;6:262.

33. English DP, Santin AD. Claudins overexpression in ovarian cancer: Potential targets for Clostridium perfringens enterotoxin (CPE) based diagnosis and therapy. Int J Mol Sci. 2013;14(5):10412-10437.

34. Casagrande F, Cocco E, Bellone S, et al. Eradication of chemotherapyresistant CD44+ human ovarian cancer stem cells in mice by intraperitoneal administration of Clostridium perfringens enterotoxin. Cancer. 2011;117(24):5519-5528.

35. Hashimoto Y, Yagi K, Kondoh M. Roles of the first-generation claudin binder, Clostridium perfringens enterotoxin, in the diagnosis and claudin-targeted treatment of epithelium-derived cancers. Pflugers Arch. 2016. doi:10.1007/s00424-016-1878-6

36. Rauh-Hain JA, Guseh SH, Esselen KM, et al. Patterns of recurrence in patients treated with bevacizumab in the primary treatment of advanced epithelial ovarian cancer. Int J Gynecol Cancer. 2013;23(7): 1219-1225.

37. Kaplan RN, Riba RD, Zacharoulis S, et al. VEGFR1-positive haematopoietic bone marrow progenitors initiate the pre-metastatic niche. Nature. 2005;438(7069):820-827.

38. Chau CH, Figg WD. Angiogenesis inhibitors increase tumor stem cells. Cancer Biol Ther. 2012;13(8):586-587.

39. Conley SJ, Gheordunescu E, Kakarala P, et al. Antiangiogenic agents increase breast cancer stem cells via the generation of tumor hypoxia. Proc Natl Acad Sci USA. 2012;109(8):2784-2789.

40. Wagner SC, Ichim TE, Ma H, et al. Cancer anti-angiogenesis vaccines: Is the tumor vasculature antigenically unique? J Transl Med. 2015:13:340. doi:10.1186/s12967-015-0688-5 